\title{
Hamlet ou le désir de stratégie
}

Jean-Paul Charnay

\section{(2) OpenEdition \\ Journals}

Édition électronique

URL : http://journals.openedition.org/shakespeare/1215

DOI : $10.4000 /$ shakespeare. 1215

ISSN : 2271-6424

Éditeur

Société Française Shakespeare

Édition imprimée

Date de publication : 1 novembre 1990

Pagination : 101-112

Référence électronique

Jean-Paul Charnay, "Hamlet ou le désir de stratégie », Actes des congrès de la Société française

Shakespeare [En ligne], 8| 1990, mis en ligne le 01 janvier 2007, consulté le 19 avril 2019. URL : http:// journals.openedition.org/shakespeare/1215; DOI : 10.4000/shakespeare.1215 


\section{SHAKESPEARE \\ ET LA GUERRE}

Ouvrage publié avec le concours de la Commission

des Communautés Européennes 
Illustration de la couverture : DERRICKE'S Image of Ireland, 1586 


\section{Hamlet ou le désir de stratégie}

La stratégie pourrait être définie comme l'actualisation du désir, au besoin en réglant les désirs contradictoires. Mais si le désir paraît absurde? Dans ce passage du présent au futur réalisant la réduction de la transcendance à l'immanence, on constate souvent que les voies stratégiques suivies mènent à des résultats insuffisants ou négatifs par rapport aux désirs, donc qu'il faut admettre la faiblesse de ses prévisions (de son intelligence); et que plus gravement encore la continuation de la lutte entraîne la mutation de ses valeurs, voire de sa personnalité. Les adversaires finissent par équilibrer leur lutte en accentuant leur ressemblance - ce qui, pour mieux réaliser la négation de l'Autre, constitue en fait une négation de soimême, débouche sur l'identification à l'Autre. Se transformant en assassin, pour justicier vengeur qu'il se veuille, Hamlet ne rejoint-il pas Claudius?

Tel est le destin dramatique - aux deux sens du terme - d'Hamlet. Car il y a la tragédie d'Hamlet; et il y a le prince Hamlet. Tel est le foisonnement shakespearien que les deux ne coïncident pas.

À la tragédie on peut appliquer les interrogations que la stratégiste est en droit de poser à l'ensemble du corpus shakespearien sur la guerre, la stratégie, la tactique, les révoltes populaires, la géopolitique et la souveraineté. Non certes que ces thèmes y apparaissent plus qu'en d'autres pièces: au contraire. Mais leur présence parfois infinitésimale dans la tragédie d'Hamlet permet, en travaillant aux limites, de mieux tester leur essence et leur rôle. Inversement la richesse du caractère d'Hamlet débouche sur une philosophie de la stratégie. 


\section{La guerre dans Hamlet}

On précisera quelques points : typologie des combattants, morphologie des batailles, géopolitique, principes de la guerre.

- Typologie des combattants; plus largement, psycho-stratégie des protagonistes, c'est-à-dire classement par attitude, par caractère des personnages en rapport avec la guerre dans le drame.

Dans l'éblouissant éventail offert par le corpus shakespearien, Hamlet demeure assez marginal. Pas de grands rois capitaines (Henry V, Jules César, Richard III...). Pas de grands généraux, qu'ils soient machines de guerre, montagnes de muscles aux systèmes nerveux fragiles (Othello, Marc-Antoine, Macbeth en partie...), ou héros quasi homériques (Talbot ...). Pas de truculents et éblouissants sous-ordres tels Domitius Aenobarbus ou Falconbridge ...). Pas de grands courtisans soldats tels que ceux des cours des Tudors (Sidney, Raleigh...) ou des Valois (Bussy d'Amboise selon Chapman), modèles idéalisés par Balthazar Castiglione et analysés par Balthazar Gracian, et tel qu'aurait pu le devenir Laertes s'il avait vécu; au contraire le sombre doublet Rosenkrantz Guildenstern et le futile Osric apparaissent négatifs de ce modèle du héros courtisan. Encore moins d'officiers subalternes capitaines ou lieutenants (Iago, Cassio...) car Bernardo, Marcello... ne sont dans la pièce que des utilités, non des caractères. Ils marquent cependant que l'on est passé de l'âge des chevaliers (suffisance et décrépitude de la chevalerie française avant Azincourt dans Henry $V$ ) à celui des soldats de métier, des capitaines de fortune. Mais pas de miles gloriosus tels Falstaff et ses commensaux basofficiers. Nym, Pistol et Bardolph.

$\mathrm{Ne}$ restent donc pour la typologie des combattants que le jeune héros guerrier, Fortinbras, mais moins profond, moins philosophe que Hotspur - peut-être parce que réussissant il s'installe dans l'histoire, tandis que ce dernier voit fuir pour lui le temps... Et, en cette période où, en dépit d'Elisabeth, la royale est d'abord une 
fonction guerrière, ce jeune roi capitaine succède aux deux rois déchus qui ne se réfugient ni dans la sérénité tel Prospero, ni dans la folie tel Lear, ni dans la mort tel Richard II, ni dans les intrigues et les compromis tel Jean sans Terre, mais dans leurs regrets respectifs: en clair le père d'Hamlet; en sombre, le vivant n'assumant pas ses remords et poursuivant pourtant ses crimes: Claudius Restent Hamlet et Horatio: nous y reviendrons.

- Morphologie des batailles. Elle apparaît également très peu dans Hamlet: le combat du vaisseau contre les pirates, le début de sédition vite calmée occasionnée par le retour de Laertes, et la campagne polonaise de Fortinbras : tout cela est à peine évoqué.

La carence est peut-être moins importante qu'il ne semble, car il est curieux de constater qu'en dépit du nombre prodigieux de guerres qu'offre le théâtre de Shakespeare, la description des combats renvoie peu souvent à l'ordre des batailles, à la disposition respective des armées et à la logique de leur déroulement, tant que cette logique n'est précisément pas encore atteinte par ce déroulement qui débande les troupes organisées et dissout les grands chocs collectifs en combats singuliers. En fait le théâtre de Shakespeare «visionne» la fin des batailles: lorsque les mêlées confuses ont fragmenté les hosts et les compagnies, les dissolvent en des affrontements de petits partis, en corps-à-corps qui se terminent en duels ou en curée (la mort de Richard III).

Certes la guerre entraîne la mort, et dans les mises en scènes les idéologies pacifistes actuelles ont beau jeu de démystifier celle-là en montrant celle-ci en gros plans, au ralenti. Mais Callot dans les Malheurs et Goya dans les Désastres de la guerre ont été bien plus forts. Et l'on pourrait à l'inverse à partir du texte même insister sur la conception de la bataille: sur la stratégie militaire.

Force est cependant de reconnaître que eu égard aux conflits historiques, mis en scène, (fin de la guerre de Cent ans, guerre des deux Roses...) et dont les récits sont tirés des historiens de l'époque, c'est surtout la guerre de la féodalité sur son déclin qui est décrite. Guerre de chevau- 
chées, de rencontres de cavalerie, d'attaques frontales, de chevaliers en armures, donc de fait d'armes, de trêves, de combats singuliers qui sont presque des jugements de Dieu (Jeanne et Talbot, Macbeth et Macduff...): ce que devient le duel Hamlet-Laertes. En dépit de Plutarque cette conception réagit en partie sur les pièces romaines, Jules César, Antoine et Cléopâtre, Coriolan.

Or la Renaissance joue aussi en matière militaire. Les princes et les condottieri se font traduire et commenter les traités de guerre et les historiens grecs, latins et byzantins par les érudits. Et à l'époque de Shakespeare le renouvellement de l'art militaire conjoint la centralisation et la «mercenarisation» des armées par les puissantes monarchies face à l'host médiéval, le progrès technique de l'artillerie, et des interrogations sur la phalange grecque, la légion romaine. Au long des guerres de Bourgogne, d'Italie, et de Flandre, des guerres de religions et des guerres nationales par lesquelles les Valois de France dessèrent l'étreinte des Habsbourgs autrichiens et espagnols, s'effectue une mutation fondamentale de l'art militaire et des ordres de batailles: de la cavalerie blindée à la bataille suisse, bloc monolithique de piquiers, puis au tercio espagnol articulant piquiers et mousquetaires. Or cette grande mutation n'est guère prise en compte dans Shakespeare, ni dans les pièces historiques, ni dans les pièces «intemporelles» dont Hamlet théâtre de «légende» plus que de chronique, fait partie. Claudius certes appelle bien ses Suisses à la rescousse; mais après la guerre de Cent ans les Anglais perdus dans leurs guerres civiles apparaissent peu sur les champs de bataille continentaux.

Shakespeare sans doute semble avoir eu quelques connaissances tactiques du combat (on a voulu en faire un sergent d'armes d'infanterie, un quartier maître de marine); il a mis en scène les matériels: archers, arbalétriers, artillerie - avec anachronisme dans le le Roi Jean et Hamlet. Mais globalement la guerre dans Shakespeare consiste davantage en des combats de rencontre, stratagèmes, alarmes, escarmouches, sièges de châteaux ou de 
villes, se cristallisant après de longues chevauchées qu'en des opérations militaires longuement élaborées ou des projections et réorganisations continentales ou impériales très conceptualisées comme par exemple dans Nicodème ou Mithridate. On ne parle évidemment pas ici de la grande politique si souvent débattue avec force par Shakespeare, mais de sa vision de l'«art de la grande guerre» qui évoque plus celui des Chroniques de Froissart

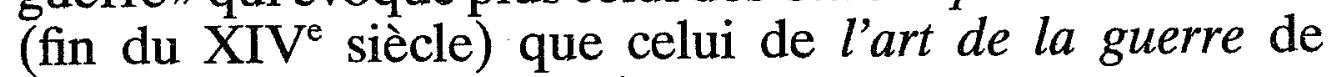
Machiavel (début du XVI ${ }^{\mathrm{e}}$ ).

Apparaît peu également au-delà de la rhétorique patriotique - combien splendide : «cette île sertie dans une mer d'argent... - une évocation tactique, opérationnelle, de la montée en puissance navale alors réalisée par l'Angleterre des Tudors: de la grande nef de Henri VIII pieusement restaurée au musée naval de Portsmouth aux grands navigateurs d'Elisabeth: Drake, Raleigh... Dans Hamlet le combat contre les pirates demeure un classique abordage loin du grand choc d'Actium dans Antoine et Cléopâtre. Dans Othello la flotte de guerre ottomane dont la menace sur Chypre motive l'envoi immédiat d'importants renforts et du meilleur de ses généraux par la Sérénissime s'évanouit dans l'espace marin. Sans doute n'était-ce pas le sujet de la pièce, mais la puissance navale ottomane n'avait pas été totalement brisée par Lépante, et une escadre de raid n'était point équivalente à cette lourde flotte de charge composée de bâtiments hétéroclites et certains fort peu marins, qu'était l'Invincible Armada.

Plus profondément peut-être que la bataille navale, la circumnavigation à valeur d'initiation ou de contreinitiation (guerrière pour Henri $\mathrm{V}$ passant en France, philosophique dans La Tempête, mortelle dans Hamlet par la traversée vers l'Angleterre...) dévoile l'importance de la puissance navale, elle-même élément de la géopolitique. Hamlet est loin d'être aussi riche que les pièces sur la fin de la guerre de Cent ans, relatives à la centralisation par les grands royaumes se transformant en ÉtatsNations, ou les pièces romaines évoquant la géopolitique impériale et les fondamentales ruptures et interférences 
culturelles et stratégiques entre Orient et Occident (Antoine et Cléopâtre, Jules César...). Pourtant par la guerre de Pologne apparaît cette errance, cette incertitude des frontières qui va devenir l'une des constantes géopolitiques des conflits entre peuples germains, scandinaves et slaves dans l'Europe médiane. Errance des frontières qui renvoie à la conduite de la guerre, et à ses principes.

-Principes de la guerre. Les principes de la guerre se placent au-dessus de la morphologie de la guerre, c'est-àdire au-dessus de la stratégie, de la tactique et de l'évolution des armements. Ils constituent des attitudes générales de l'esprit, des orientations de l'action, que politiques et capitaines doivent considérer lorsqu'ils établissent leurs plans concrets, lorsqu'ils les adoptent, les transforment, les renient parfois en fonction de l'évolution du conflit. Leur énoncé demeure fluctuant, et c'est souvent par synthèse a posteriori que les théoriciens et les commentateurs les dégagent des précédentes campagnes pour en inspirer les campagnes futures.

Il serait donc légitime de rechercher leur formulation plus ou moins explicite à travers le corpus shakespearien, et d'en tirer une architecture conceptuelle que l'on pourrait comparer à celle proposée par les traités en formes, soit relativement contemporains (par exemple: les Instructions sur le faict de la guerre de Guillaume du Bellay, 1548), soit postérieurs (Les principes de la guerre de Foch).

Il semble que l'analyse serait très riche. Par exemple - simple sondage - dans le Roi Jean (IV.ii), ou dans Richard II (II.ii) sont pris en compte les principes d'initiative et de célérité : d'une avance dans le temps, donc de concentration des forces. Encore faudrait-il déterminer si cette richesse résulte d'une connaissance particulière de Shakespeare ou si elle coïncide avec un savoir culturel commun aux politiques et aux capitaines de ces périodes guerrières.

L'ensemble de ces analyses typologiques, morphologiques et axiomatiques exigerait l'élaboration d'une 
thématique lexicale et conceptuelle et un traitement informatique. Alors se décanterait avec une ampleur la vision shakespearienne de la conduite de la guerre.

Resterait à en préciser son essence et sa fonction. Mais là, au-delà de la tragédie d'Hamlet intervient la personnalité, le caractère et les réactions du prince Hamlet.

\section{Hamlet et la stratégie}

Aboulique et masochiste tel est-il souvent présenté. Que ces portraits réfèrent à la pyschanalyse (selon Ernest Jones, atteint du complexe d'Oedipe il n'arrive pas à détruire Claudius parce que celui-ci à précisément fait ce qu'Hamlet désirait faire : tuer le père et posséder la mère), ou au remords chrétien (le spectre de son père n'est-il pas en vérité infernal, leurre pour le perdre?), ou au scrupule judiciaire (les preuves surnaturelles sont-elles suffisantes pour justifier la répression?), ou ... (puisque les interprétations surabondent): peu importe pour la définition d'Hamlet stratège, car il est bien conscient d'une chose : la vacuité de l'existence, de ses aspects mécaniques.

Cette conscience de l'absurdité du fait de vivre annihile-t-elle vraiment en lui, comme il a souvent été soutenu, tout désir de stratégie? Rien n'est moins sûr. Car d'où vient chez Hamlet cette forte conscience - qui n'est pas fatalement pessimisme - de l'absurdité de la vie? Vraisemblablement des traumatismes que lui infligent les quatre amours de sa vie: - son amour pour Ophélie : qu'il la désire charnellement ou qu'il soit, selon certaines interprétations, plus attiré par une amitié particulière avec Horatio importe peu car prince puis roi, et quels que soient ses goûts sensuels il saurait avec elle assurer la descendance de la dynastie. D'autre part, en deçà de la sexualité il est sensible au charme, au raffinement, à la gaieté tendre de la jeune fille. Mais lors de la scène (après le trop fameux monologue) où Ophélie, manipulée par son père et Claudius, fait semblait de lire pour le 
rencontrer, et qu'il lui demande où se trouve son père, elle répond «at home» alors qu'elle sait pertinemment qu'il est caché derrière la tapisserie, il la prend en flagrant délit de tromperie. Que ce soit par motifs pieux, par utilité sociale, par amour pour lui, la pure Ophélie ment. Et Hamlet ne peut le lui pardonner ${ }^{1}$. D'où le redoublement vrai ou simulé de sa colère, accentuation de son côté «macho» déjà à vif par la vision de l'acte sexuel commis par sa mère avec le frère meurtrier de son père («o fragilité, ton nom est femme»...) et qui le pousse à brutaliser la jeune fille, à lui lancer des plaisanteries incongrues avant la représentation des comédiens, mais plus profondément qui exacerbe sa dérision de Polonius et de Claudius se cachant comme des valets et qui le pousse vers l'action directe violente.

- Son amour des livres. Hamlet à Wittenberg: que n'a-t-on glosé sur l'influence brumeuse de la philosophie allemande? Mais il est indéniable qu'il est très «intellectuel», au bon et au mauvais sens du terme. Raffinant sur la poésie avec les comédiens, mesurant ses concetti avec le fossoyeur, évoquant avec émerveillement le subtil bavardage d'Yorick, maniant le paradoxe absurde contre la logorrhée diplomatique de Polonius ${ }^{2}$ et la rhétorique spécieuse avec les courtisans, procédant en dernière analyse à une déconstruction syntaxique: «words, words, words...»

Plus essentiellement, trop philosophe, il a une conscience aiguë moins du tragique que de l'absurde des choses de la vie, de la médiocrité de la politique, et il a compris qu'un roi n'a point à être philosophe, c'est-à-dire un contempleur critique de la réalité et des apparences, pas plus qu'un philosophe n'a à être roi, c'est-à-dire un maître des stratagèmes et des violences. Trop bonne cartésienne, la reine Christine abdiquera...

Mais lettré élisabéthain, Hamlet est aussi, tel Sir Philip Sidney, un héros, un prince accompli de la Renaissance. Le prouve - Son amour des armes. Courageux, l'épée à la main? Au-delà Hamlet professe une vraie passion pour l'escrime. Il est le premier à se lancer à 
l'abordage sur le vaisseau pirate, et ne peut revenir à son bord. Au reste si Ophélie est trop raffinée pour être séduite comme la petite Vénitienne, Desdémone, par de simples récits de batailles, par les pompes et exploits militaires - «pomp and circumstance» selon l'expression d'Othello - elle est inversement trop équilibrée, trop dame de la cour, trop «dans le siècle», au grand sens du terme, pour aimer un autre qu'un cavalier capable de manier l'épée avec autant de brillant que d'esprit.

Aussi lorsque le roi organise le challenge avec Laertes, challenge présenté comme un simple jeu amical, Hamlet ne se dérobe pas. En dépit de ses pressentiments funestes des conseils d'Horatio, il relève le défit. Évident goût des armes - mais plus profondément prescience que la violence en principe muselée, mouchetée, de l'affrontement, se déchaînera; et qu'ainsi dopé il parviendra à ce qu'il n'a pas encore réussi à faire: surmonter ses inhibitions et tuer Claudius? Claudius lui-même qui, par raison d'État (à l'évidence Hamlet avec sa soif de vengeance est devenu aussi dangereux pour la stabilité du pouvoir, la légalité établie et la paix du pays que l'était Antigone), transforme par fourberie en traquenard l'assaut d'armes qui à son tour se transforme en duel judiciaire, en ordalie: en jugement de Dieu déterminant le massacre de la dynastie régnante. À leur échelle respective la lutte de François Ier et Henri VIII au Camp du Drap d'or, ou le duel de Jarnac et La Châtaignerie, avaient montré les conséquences redoutables de tels affrontements pour le prestige des souverains et de leurs dynasties... Dernier amour d'Hamlet:

- Cet amour de sa dynastie: du pouvoir transmissible de la majesté, de la souveraineté danoise. Amour caché et exacerbé dans la pièce par ses rapports avec ses parents dégradés: son père assassiné hors de l'état de grâce, sa mère séduite et polluée par le meurtrier de son mari telle Lady Ann par Richard III.

Hamlet certes comprend fort bien que l'analyse des raisons d'agir dissout la raison - et non seulement la volonté - d'agir: son suicide manqué, ses hésitations à 
tuer. Hamlet, cet Oreste sans Electre et sans Hermione ou plutôt dont l'Hermione est la trop douce Ophélie, n'arrive pas à tuer son Égisthe - le roi Claudius - et encore moins sa Clytemnestre - la reine Gertrude: il refuse expressément d'être Néron se sacrifiant Agrippine.

Pourtant Hamlet se révèle le prince - le roi durant quelques instants avant sa mort - qui assure par une épuration de grand style, par un massacre paroxysmique (son oncle et sa mère, toute la famille du grand chambellan Polonius, et les courtisans spécieux Rosenkrantz et Guildernstern) le passage de la vieille dynastie «pourrissant le royaume» à une dynastie nouvelle, vigoureuse : Fortinbras le Norvégien.

Paradoxale dialectique donc qu'agit Hamlet: son sentiment aigu de l'absurdité de l'action résultant de son propre déphasage familial et royal lui fait imaginer des processus machiavéliens: ainsi du meurtre «spontané», par réflexe de Polonius, en voulant faire croire qu'il croyait que c'était le roi; ainsi de la pantomine/jeu de miroir des acteurs; ainsi de la ruse (un faux) par laquelle il substitue Rosenkrantz et Guildernstern à lui-même pour être tués en Angleterre; ainsi de la naïveté captieuse du plaidoyer à Laertes sur les morts de Polonius et d'Ophélie: «ce n'est pas Hamlet...». Prince des faux-semblants il sait qu'il ne pourra plus être un prince juste, le «prince chrétien » selon Érasme.

Pourtant il demeure intègre, inentamé: il n'est point gangrené par sa «folie» aussi profondément que Lorenzaccio dissous jusqu'aux mœlles par sa débauche d'abord simulée. Il ne se transforme pas en un monstre, en un prince machiavélique, tels César Borgia, Louis XI ou Richard III. Refusant d'être un roi de ruse, un acteur de real-politik comme Créon, comme Égisthe, mais se reconnaissant lui-même comme membre pourri du royaume de Danemark, il récuse sa propre royauté.

Admettant, comme les Anglais de l'époque de Shakespeare la guerre comme une sorte de jugement de Dieu permutant les dynasties (alors qu'à la même époque les Français font la guerre pour ne pas changer de dynas- 
tie), il transmet la couronne au guerrier revenu victorieux des plaines de Pologne, à un jeune roi-capitaine qui agira selon une politique militaire et juste, avec comme conseiller le seul esprit équilibré du drame: «Tu es un homme, Horatio...».

Rationalité suprême, stratégie absolue donc que celle d'Hamlet qui se détruisant en tant que roi et en tant qu'individu se précipite dans la mort. Incarnant, pendant les quelques instants où le poison coule en ses veines la souveraineté du royaume, «étant» la majesté danoise, il se nie lui-même comme il a nié Claudius et reconduit, en politique à la fois réaliste et juste, la continuité de l'État par un changement de dynastie.

Non sans une secrète ironie: héros qui aurait su se bien conduire en bataille, il tempère son admiration pour Fortinbras agissant par la considération de l'absurdité en soi de guerres menées pour des «lopins de terre» et le pressentiment que Fortinbras se lancera peut-être dans des aventures militaires aussi extravagantes que celles de Charles XII entraînant sa perte, le déclin de son royaume. Car c'est non seulement l'absurdité du présent qui dissout les raisons d'agir, donc le désir de stratégie, mais aussi l'absurdité des futurs possibles.

Pourtant il préfère l'action fut-elle à objectif dérisoire mais poursuivie dans et pour l'honneur à cette entropie de la volonté, à cette pensée oscillante qui réduit les opportunités à «prendre le nom d'action».

Ainsi Hamlet, prince des faux-semblants, demeure cependant un être social, un prince politique pour qui la guerre, en dépit de son absurdité, demeure un critère de validité, de compétence et de légitimité. Se situant dès lors au-delà de l'absurde, même s'il en demeure persuadé, il récupère son désir de stratégie. Ses quatre amours meurtris l'apaisent par leur réunion dans son amor fati, qui transcende même l'absurde de sa destinée qu'il ne peut cependant dépasser une fois cette destinée accomplie : «le reste est silence».

Pourtant Horatio ni Fortinbras ne s'y trompent, le 
prince Hamlet est mort en soldat, est mort en roi assurant les hautes charges de la majesté souveraine: la justice par la force et la perpétuation de la légitimité du pouvoir. Métaphysiquement et non seulement théâtralement il doit être honoré par des funérailles guerrières et royales. Au-delà des innombrables et contradictoires interprétations du personnage, les mises en scène de la pièce convergent toutes dans la vision finale qui réassemble la tragédie d'Hamlet et le destin du prince Hamlet; le roi Hamlet sort de sa vie, sort de scène sur un pavois porté par quatre capitaines. C'est son «silence» qui fait éclater les fanfares et les salves qui l'insèrent dans l'histoire et, par une dernière absurdité stratégique, dans une longue lignée de rois guerriers....

\author{
Jean-Paul CHARNAY \\ Directeur du Centre d'Étude et Recherche sur les \\ Stratégies et les Conflits, CNRS. Sorbonne.
}

\title{
NOTES
}

(1) Encore Hamlet ne voit-il pas Ophélie folle, chantant d'impudiques paroles. Mais pourquoi? Déplore-t-elle tant la mort de son père, ou comprendelle qu'elle ne pourra jamais épouser le meurtrier de son père? Alors ressortirait toute sa frustration de femme qui ne sera pas possédée par l'aimé - Antigone le disait avec moins de charme déchirant mais plus de noblesse. Chimène au contraire, loin de perdre la raison ou de se lamenter, se transforme en plaideuse acharnée à se faire prouver qu'en dépit de ses poursuites elle pourra quand même épouser Rodrigue...

(2) Il y a du M. de Norpois en Polonius. 